Historic, Archive Document

Do not assume content reflects current scientific knowledge, policies, or practices. 



\section{TO THE TRADE}

We take pleasure in presenting this, our final wholesale list of the Spring season. We urge you to use same as often as you are in the market for Gladiolus Bulbs.

New customers are always welcome. Do not hesitate to send small orders, for we know from past experience that the small order of today is the large order of tomorrow. We will give the same personal attention, whether the order is for $\$ 5.00$ or $\$ 500.00$.

Every order received will be treated as a rush order, muless otherwise instructed. We are prepared to handle many orders per day and can make shipment on each and every item herein listed within twenty-fotr hours of receipt of order, as lone as available stocks last. We are sure our trade friends will appreciate this service in the rush season. To save confusion and extra correspondence mention second choice in ordering if you have same.

PREP.IRE FOR A PROSPEROUS SURING SEASON by liberally stocking itens you neerl. Iispecially he sure you have enough of such splendid novelties a INNIE L. ALRIE. MIRS. LEON DOUGLASS, OR.INGE QUEEN, IV. H. PHI'PS, DR. F. E. BENNETT, PITTEER'S TRIUNPH. HOLLYHOCK, YVONNE, P.IUL, PFITZER and VEILCHENBLAL. Yonr customers want the new and better varieties. Keep then satisfied with plenty of noveltics. I'T WILI, P.IS JOU. The large sizes are short crop this season due to the drought last summet.

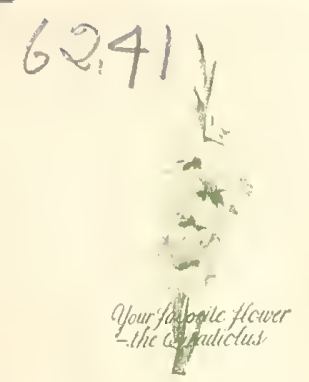

\section{HUNTER'S April 1930 Trade List}

Charles E. F. Gersolorf

1825 No. Cepitol st.

Washington, D. C.

POSTMASTEK:

If Unable to Deliver, Return Postage Guaranteed

By GEORGE W. HUNTER-Grower

R. F. D. No. 4, Dowagiac, Mich.

\author{
Ser. $43,5 ! 2$ I. L. \& R. \\ I. S. POSTAGE \\ P A I D \\ I)OWAGIC, MICH. \\ Permit No. 12
}

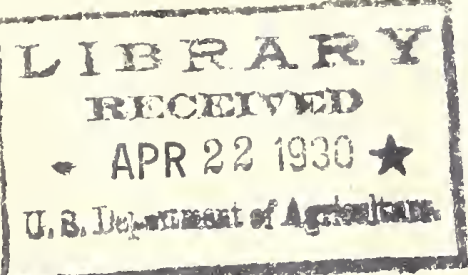

\section{Terms and Conditions of Sale:}

With these prices all previous quotations are withdrawn.

All Bulbs are sold f. o. b. Dowagiac, Michigan, and travel at risk of purchaser.

All Bulbs offered in this list are healthy, true-to-name, and strictly up to size. We do not substitute without permission. Mention second choice in ordering if you have same. your order.

Delivery on demand. We ship daily. If you want a rush order designate same on

All bulbs are offered subject to prior sale and to any or all contingencies beyond our control.

$5 \%$ discount cash with order; $2 \% 10$ days; net 30 days. Gash with order or satisfactory references required from unknown correspondents. 
R. F. D. No. 4, Dowagiac, Mich.

\section{Pink and Rose-Pink Hues}

America (f), mid-season

ANNIE LAURIE (f), late mid-season

APPLEBLOSSOM, new, tall, late

Crinkles Extra Ruffled Pink

Dr. IV. Van Fleet (f), tall, early

E. J. Shay'lor (f), ruffled, second early

Evelyn Kirtland, Tall Pink

Le Marechal Foch (f), large, early

LONGFELLOW, new, mid-season

Mona Lisa, ruffled, mid-season

MRS. DR. NORTON (f), early mid-season

MRS. FRANK PENDLETON (f), 1. m.-sea.

MYRTLE (f), dainty, early

1910 Rose (f), early

Panama (f), late

Pink Wonder, mammoth, mid-season

Rose Glory, ruffled, mid-season

Rosemary, novelty, mid-season

Wilbrinck (f), extra early

Youell's Favorite, dependable, late
Eugenc Lcefebreve, blotched, extra

Miss Madison, large, late

Ruffled America, ruffled, late mid-season

Per 100 Bulbs

No. 1 No. 2 No. 3 No. 4 No. 5 No. 6 Withdrawn for Season-Sold Out

$\begin{array}{rrrrrr} & 12.00 & \$ .00 & \$ 6.00 & \$ 4.00 & \$ 2.50 \\ & & & 2.25 & 1.50 & 1.00 \\ & & & +.00 & 3.00 & 2.0 \\ 3.00 & & & 1.50 & & \\ & & & 1.25 & 1.00 & .2 .40 \\ & 2.40 & 1.75 & & & \end{array}$

IIthdrawn for Season-Suld Out

$\begin{array}{lllll}2.50 & 1.50 & 1.00 & .75 & .50\end{array}$

Withdrawn for Season-Sold Out

$\begin{array}{lll}4.00 & 2.25 \quad 1.50\end{array}$

$3.00 \quad 2.40$

$\begin{array}{lllll}3.00 & 2.25 & 1.50 & 1.25 & .75\end{array}$

Withdrawn for Season-Sold Out

$\begin{array}{llllll}4.00 & 3.00 & 2.25 & 1.50 & 1.25 & .75\end{array}$

$\begin{array}{ll}2.50 & 1.00\end{array}$

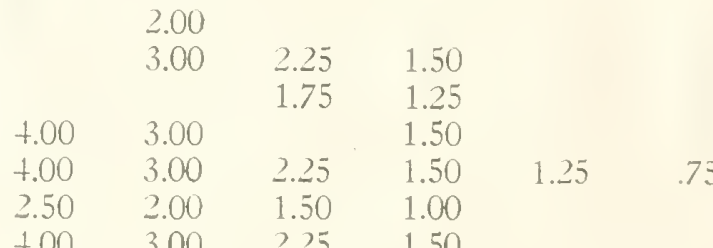

\section{Salmon-Pink, Orange, Orange-Pink Hues}

Alice Tiplady (f), second early

Fay Lanphier-Large

Gretchen Zang (f), standard late

Halley (f), extra early

Jean du Tailles-Late, extra

LOS ANGELES (f), second early

Maidensblush (f), extra early

MARSHALL FOCH (K), mid-season

MRS. H. E. BOTHIN, ruffled, late

MRS. LEON DOUGLASS- Mam. Novelty

Nancy Hanks-Orange Pink

$\operatorname{Odin}(\mathrm{f})$, second early

ORANGE QUEEN (f), second early

PHAENOMEN, novelty, early

Primunella, (f), ruffled, second early

P'rince of Wales, popular, early

Salmon Beauty (f) second early

Tycko Zang, late mid-season

VIRGINIA HALE, late mid-season

W. H. PHIPPS, novelty, late

Zona (f) Novelty Prim

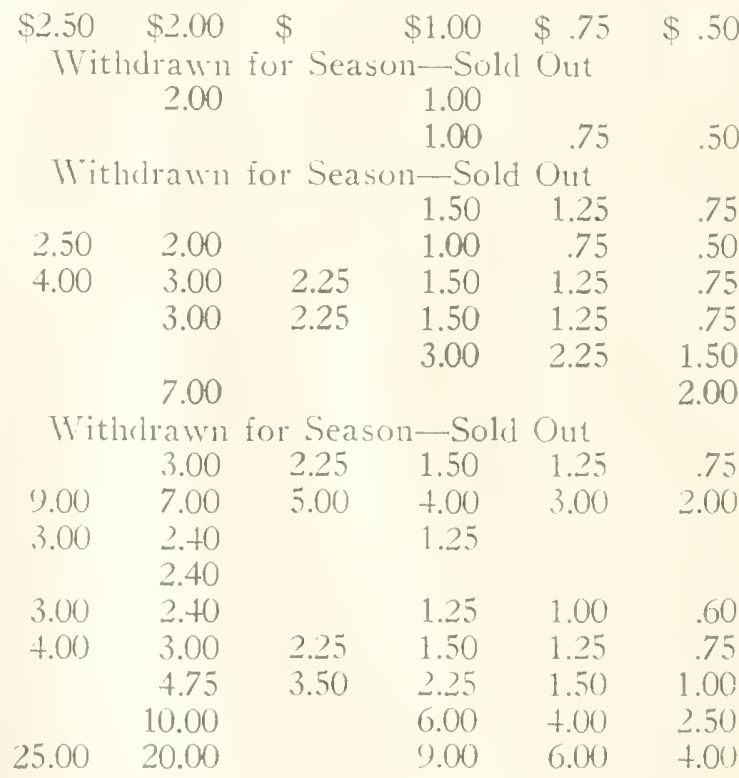

\begin{tabular}{|c|c|c|}
\hline 25 Bulbs & The Following Quantity Rates Will Apply: & Bulblet Prices on Application. \\
at the & 250 Bulbs at twice the 100 rate. & Sutate Your Exact Wants. \\
100 Rate. & 1000 Bulbs at 8 times the 100 rate. & States rate.
\end{tabular}




\section{GEORGE W. HUNTER-Grower}

R. F. D. No. 4, Dowagiac, Mich!

Mechanically Graded, Hand Selected Stock

\section{Dark Red, Red, Scarlet, Orange-Red Hues}

CARUSO (f), novelty, early

No. 1

CHATEAU THIERRY (f), mid-season

Crimson Glow (f), mid-season

DR. F. E. BENNETT, mid-season

Firy Knight, early mid-season

Goliath-Dark Red

HERBSTZAUBER, novelty, late

K.IRL VOLKERT (f), extra early

KRE1.AGE'S FAVORITE, cxtra early

Mrs. Francis King (f), standard late

PERSIA, black-red, mid-season

PFITZER'S TRIUMPH, new

Rajal, mid-season

SCARLET PRINCEPS (f), mid-season

Scarlet Cardinal-Landscape Prim

Yer 100 Bulbs

No. 2 No. 3 No. 4 No. 5 No. 6

$\$ 7.00 \quad \$ 5.00 \quad \$+.00 \quad \$ 3.00 \quad \$ 2.00$

$\begin{array}{rrrrrr}\$+.00 & & 2.25 & 1.50 & 1.25 & .75 \\ & & & 4.00 & 3.00 & 2.00 \\ 4.00 & 3.00 & 2.25 & 1.50 & 1.25 & .75 \\ & 3.00 & 2.25 & 1.50 & 1.25 & .75 \\ 15.00 & 12.00 & 9.00 & 6.00 & 4.00 & 2.75 \\ 6.00 & +.75 & 3.50 & 2.25 & 1.50 & 1.00 \\ 6.00 & 4.75 & & 2.25 & 1.50 & 1.00 \\ 2.50 & & 1.50 & 1.00 & .75 & .50 \\ 6.00 & +.75 & 3.50 & 2.25 & 1.50 & 1.00 \\ & 80.00 & 60.00 & +5.00 & 30.00 & 20.00 \\ & 7.00 & 5.00 & +.00 & 3.00 & 2.00 \\ & & 1.75 & 1.25 & 1.00 & .0(1) \\ 4.00 & 3.00 & & 1.50 & 1.25 & .75\end{array}$

\section{Mauve, Lavender-Blue, Light Violet Hues}

BYRON L. SMITH, second early

Blue Orchid-Mid-Season

CAPT. BOYNTON, (f), large, mid-season

Catherina, (f), tall, early mid-season

Geraldine Farrar-Lavender-Violet

Herada (f), mid-season

HEAVENII' BLUE, novelty, each

Jane Addams-Extra Large

Magic-Extra Early

Mary Fennell, second early

MAD. von KONIJNENBURG, novelty

MRS. F. C. PETERS (f), late

Ocellus, rare novelty, early micl-season, each

Rev. Ewbank (f), early

RUTH HUNTINGTON, late mid-season

\section{Cream, Buff, Yellow Hues}

Fortuna, novelty, extra early

GOLD EAGLE, (f), extra early

GOLDEN FRILLS, (f), early

GOLDEN MEASURE, (f), mirl-season

Mary Stearns Burke, late

Ming Toy, (f), early.

Morning Glory-Mid-Season

Niagara, late mid-season

Obelisque-Large Novelty

Pfitzer's Yellow Wonder-Second Early

Schwaben, (f), standard late

SOUVENIR, (f), early

Starbright, (f), second early

Topaz, (f), early 1'er 100 Bulbs except as noted

$\begin{array}{lrrrr} & \$ 2.25 & \$ 1.50 & \$ 1.25 & \$ .75 \\ 4.75 & 3.50 & 2.25 & 1.50 & 1.00 \\ +.75 & 3.50 & 2.25 & 1.50 & 1.00 \\ 3.00 & 2.25 & 1.50 & 1.25 & .75\end{array}$

Withdrawn for Season-Sold Ont

$\$ 3.100 \quad 1.25$

$\begin{array}{llllll}5 .(0) & +.0(0) & 3.25 & 2.50 & 1.30 & 1 .(1)\end{array}$

Withelrawn for Seasun-Solel Ont

$\begin{array}{llllll}0.00 & 4.75 & 3.50 & 2.25 & 1.50 & 1.0(1)\end{array}$

$3.00 \quad 1.50$

$80.00 \quad 60.00 \quad+5.00 \quad 30.00 \quad 20.00$

$\begin{array}{lllll}+.75 & 3.50 & 2.25 & 1.50 & 1.00\end{array}$

$\begin{array}{llllll}3.00 & 2.50 & 1.75 & 1.25 & 1.00 & .75\end{array}$

$\begin{array}{lll}4.75 & 3.50 & 2.25\end{array}$

$\begin{array}{llll}+.75 & 3.50 & 2.25 & 1.50\end{array}$

1.00

\begin{tabular}{|c|c|c|}
\hline 25 Bulbs & The Following Quantity Rates Will Apply: & Bulblet Prices on Application. \\
at the & 250 Bulbs at twice the 100 rate. & State Your Exact Wants. \\
100 Rate. & 1000 Bulbs at 4 times the 100 rate. & times the 100 rate.
\end{tabular}


GEORGE W. HUNTER-Grower

R. F, D. No. 4, Duwagiae, Mich.

Mechanically Graded, Hand Selected Stock

\section{Cream, Blush, White Hues}

Carmen Sylva, mid-season

linopa-iate Exhibition

FERN KYLE, (f), ruffled, mid-season

HOLLYHOCK, ruffled, early mid-season

Imperator, late, mid-season

L'Immaculee (f), early mid-season

Lily White, (f), early

Lidy, early

MAINE, tall, mid-season

MARIE KUNDKED (f) rutfled, carly

Mary Pickford, (f), popular early

Peace, (f), standard late

Vesta Tilly, (f), early

White Beauty-Early lixhibition

TONNE (f), Exccllent, early.

\section{Violet-Blue, Violet, Purple Hues}

Anna Eberius, mid-season

BARON J. HULOT-Violet-Purple

Henry Ford, mid-season

J.1COB $\triangle$ van BEIEREN, mid-season

PAUL PFITZER, ruffled, early mid-season

I'URPLE GIORT-Popular Ruffled

Purple Perfection, late mid-season

VEILCHENBLAU, early mid-season

Violet Glory, second early

\section{Rose-Red, Tyrian and Smoky Hues}

Chocolate Queen-New, Extra

Dr. Nelson Shook-Tyrian Rose

Emile Auburn-Smoky Novelty

John T. Pirie-Almost Brown

Mrs. Geo. W. Moulton, rose-red, mid-sea.

Mrs. William Kent, novelty, late mid-sea.

ROMANCE, (f), smoky, mid-season

Rose Ash, smoky, mid-season

Taro-American Beauty
Per 100 Bulbs

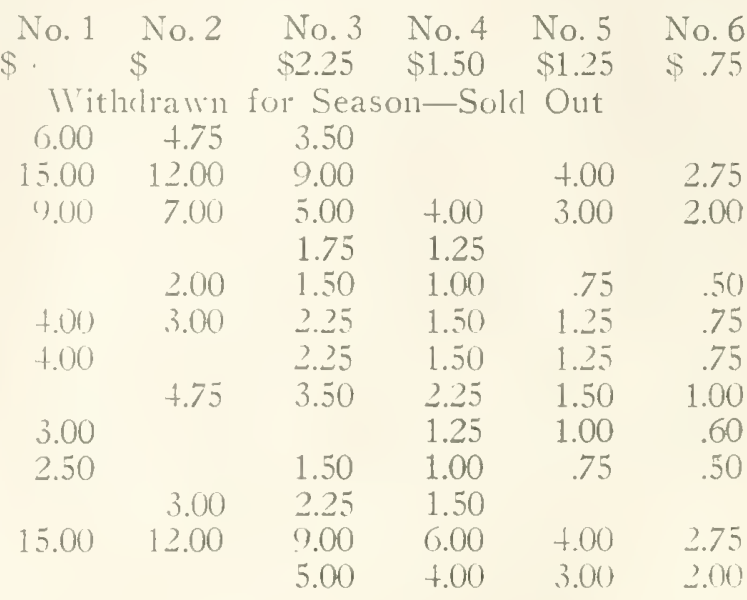

Withdrann for Season-Soll Ont

$3.50 \$ 2.25 \$ 1.50 \$ 1.00$

Tithclrawn for Season-Sold Out

$\begin{array}{rrrrrr}4.00 & 3.00 & 2.25 & 1.50 & 1.25 & .75 \\ +0.00 & 30.00 & 22.50 & 15.00 & 12.50 & 7.50 \\ \text { Withdrawn } & \text { for Season-Sold } & \text { Out } & \\ 4.00 & 7.00 & 5.00 & t .00 & 3.00 & 2.00 \\ (0.00 & & 35.00 & 22.50 & 15.00 & 10.00 \\ & 3.00 & 2.25 & 1.50 & 1.25 & .75\end{array}$

$\$ 25.00 \quad \$ 20.00 \quad \$ 14.00 \quad \$ 9.00 \quad \$ 6.00 \quad \$ 4.00$

Withdrawn for Season-Sold Out

Withdrann for Season-Sold Out

IV ithelratrn for Season-Sold Out

$\begin{array}{llllll} & 2.40 & 1.75 & 1.25 & & \\ 4.00 & 3.00 & 2.25 & 1.50 & 1.25 & .75 \\ 4.00 & 3.00 & 2.25 & 1.50 & & \\ & 3.00 & & 1.50 & 1.25 & .75\end{array}$

Vithdratin for Season-Sold Out

\section{Bulbs \\ at the \\ 100 Rate.}

\section{Bulblet Prices on Application.} State Your Exact Wants.

IMPORTANT! Prices on all other varieties a matter of correspondence. Let us quote you on your wants. A special discount will apply on orders of 5000 or upivards of the same size and variety. We are also in position to quote attractive lot prices on large orders.

ORDER EARLY 